\title{
Identification and characterization of human uracil phosphoribosyltransferase (UPRTase)
}

\author{
Jixi Li $\cdot$ Shengdong Huang $\cdot$ Jinzhong Chen • \\ Zhenxing Yang $\cdot$ Xiangwei Fei $\cdot$ Mei Zheng $\cdot$ \\ Chaoneng Ji $\cdot$ Yi Xie $\cdot$ Yumin Mao
}

Received: 7 December 2006/ Accepted: 15 February 2007/Published online: 24 March 2007

(C) The Japan Society of Human Genetics and Springer 2007

\begin{abstract}
Uracil phosphoribosyltransferase, which catalyzes the conversion of uracil and 5-phosphoribosyl-1- $R$ diphosphate to uridine monophosphate, is important in the pyrimidine salvage pathway and is an attractive target for rational drug design by incorporation of prodrugs that are lethal to many parasitic organisms specifically. So far, uracil phosphoribosyltransferase has been reported in Arabidopsis thaliana only, not in mammals. In this study, a novel uracil phosphoribosyltransferase family cDNA encoding a 309 amino acid protein with a putative uracil phosphoribosyltransferase domain was isolated from the human fetal brain library. It was named human UPRTase (uracil phosphoribosyltransferase). The ORF of human UPRTase gene was cloned into pQE30 and expressed in Escherichia coli M15. The protein was purified by Ni-NTA affinity chromatography, but UPRTase activity could not be detected by spectrophotometry. RT-PCR analysis showed that human UPRTase was strongly expressed in
\end{abstract}

Jixi Li and Shengdong Huang contributed equally to this work.

J. Li · J. Chen · Z. Yang · X. Fei · M. Zheng · C. Ji (凹) ·

Y. Xie - Y. Mao

State Key Laboratory of Genetic Engineering,

Institute of Genetics, School of Life Sciences,

Fudan University, Shanghai 200433,

People's Republic of China

e-mail: chnji@fudan.edu.cn

S. Huang

Department of Cardiac-thoracic Surgery,

Changhai Hospital, Second Military Medical University,

Shanghai 200433, People's Republic of China

Present Address:

J. Li

Division of Biology, California Institute of Technology,

Pasadena, CA 91125, USA blood leukocytes, liver, spleen, and thymus, with lower levels of expression in the prostate, heart, brain, lung, and skeletal muscle. Subcellular location of UPRTase-EGFP fusion protein revealed that human UPRTase was distributed in the nucleus and cytoplasm of AD293 cells. Evolutional tree analyses of UPRTases or UPRTase-domaincontaining proteins showed that UPRTase was conserved in organisms. UPRTases of archaebacteria or eubacterium had UPRTase activity whereas those higher than Caenorhabditis elegans, which lacked two amino acids in the uracil-binding region, had no UPRTase activity. This means that human UPRTase may have enzymatic activity with another, unknown, factor or have other activity in pyrimidine metabolism.

Keywords Human UPRTase - Tissue distribution . Subcellular location $\cdot$ Evolutionary analysis

\author{
Abbreviations \\ UPRTase Uracil phosphoribosyltransferase \\ GFP Green fluorescent protein \\ MTC Multiple tissue cDNA \\ UMP Uridine monophosphate
}

\section{Introduction}

In organisms, purine and pyrimidine nucleotides can be generated by de novo synthesis from compounds unrelated to nucleotides and by the salvage pathways, which use nucleotides, nucleosides, or nucleobases already present in the cell (Magni et al. 1976). Uracil phosphoribosyltransferase (UPRTase, EC 2.4.2.9), encoded by the upp gene, is a key enzyme in the pyrimidine salvage pathway, which is important in nucleotide metabolism. UPRTase catalyzes the 
conversion of uracil/5-fluorouracil (5-FU) and 5-phosphoribosyl-1- $R$-diphosphate to uridine monophosphate (UMP)/ 5-FUMP and pyrophosphate in the presence of $\mathrm{Mg}^{2+}$; this is one of the reactions involved in the so-called "UMP cycle" (Magni et al. 1976; Natalini et al. (1979). UPRTase is specific for uracil, and cannot use cytosine, cytidine, deoxycytidine, or thymine in catalysis, because large exocyclic groups at position 5 of the pyrimidine base prevent binding (Carter et al. 1997; Iltzsch and Tankersley 1994). Alternatively, in higher eukaryotes uracil/5-FU can be converted to UMP/5-FUMP by the concerted action of uridine phosphorylase (UDP) and uridine kinase (UDK) (Neuhard and Kelln 1996). The orotate phosphoribosyltransferase part of UMP synthase from the de novo biosynthetic pathway also has weak UPRTase activity in mammals (Traut and Jones 1996).

UPRTases have been identified in lower eukaryotes and most microorganisms, including baker's yeast (Natalini et al. 1979), Escherichia coli (Jensen and Mygind 1996), Sulfolobus shibatae (Linde and Jensen 1996), Acholeplasma laidlawii (McIvor et al. 1983), Candida albicans (Alloush and Kerridge 1994), Crithidia luciliae (Asai et al. 1990), Bacillus subtilis (Martinussen et al. 1995), Giardia intestinalis (Dai et al. 1995), Lactococcus lactis (Martinussen and Hammer 1994), Streptococcus salivarius (Giffard et al. 1993), Saccharomyces cerevisiae (Kern et al. 1990), and Toxoplasma gondii (Donald and Roos 1995), whereas no UPRTases have been reported in higher eukaryotes, especially mammals. The amino acid sequences of UPRTases are fairly dissimilar, with overall identities ranging from 20 to $45 \%$, but with strong conservation of the active-site residues. UPRTase belongs to the structurally well-characterized phosphoribosyltransferase (PRTase) family (Musick 1981; Sinha and Smith (2001). The UPRTases from different organisms differ in their regulatory behavior. UPRTase is activated by GTP in several organisms, for example E. coli (Jensen and Mygind 1996), G. intestinalis (Martinussen and Hammer 1994), T. gondii (Schumacher et al. 2002), S. shibatae (Linde and Jensen 1996), and S. solfataricus (Jensen et al. 2005), whereas its activity seems unregulated in other organisms, for example yeast (Natalini et al. 1979) and Bacillus caldolyticus (Kadziola et al. 2002). It has been shown that CTP allosterically inhibits $S$. shibatae (Linde and Jensen 1996), S. solfataricus (Jensen et al. 2005), and yeast UPRTase activity whereas UMP, dUMP, dCMP, UDP, TTP, and dCTP allosterically inhibit yeast UPRTase activity (Natalini et al. 1979).

Several crystal structures of UPRTases have been determined, for example those of UPRTases from $B$. caldolyticus (Kadziola et al. 2002), T. gondii (Schumacher et al. 1998), and Thermus thermophilus (Kukimoto-Niino et al. 2005). All UPRTases contain a conserved thirteenresidue fingerprint region, with a core five-stranded parallel $\beta$-sheet that binds the 5-phosphoribosyl moiety of PRPP and the nucleotide product; this is termed the "PRPPbinding motif" and is characteristic for the UPRTases (Argos et al. 1983; Lundegaard and Jensen (1999). This region typically contains four hydrophobic residues then two acidic residues, two hydrophobic residues, and four small residues, for example glycine. Near the C-terminal end UPRTases contain a highly conserved sequence that is involved in uracil binding, but no sequence motif has been identified. By studying the mechanism of activation of UPRTase activity by GTP it was found that UPRTase has two forms, dimeric and tetrameric. The tetrameric form is stabilized by PRPP and/or GTP, both of which contact two dimers of the tetramer; for this reason the stimulatory effect of GTP is seen at subsaturating concentrations of PRPP only (Schumacher et al. 2002).

Previous research has shown that UPRTase was an attractive target for rational drug design by incorporation of prodrugs that are lethal to many parasitic organisms specifically. Toxoplasma gondii UPRTase binds a variety of pyrimidine analogs (Pfefferkorn et al. 1989), some of which have documented antitoxoplasmal activity. Specifically, 5-FU is converted by $T$. gondii UPRTase to the nucleotide level, where it becomes toxic to the parasite, thus serving as a subversive substrate (Carter et al. 1997). It has been shown that adenoviral-mediated transfer of $E$. coli UPRTase into colon cancer cells can enhance biochemical modulation of 5-FU; this has been used as a chemotherapeutic drug for colorectal cancer (Koyama et al. 2000).

The hypothetical human protein with a uracil phosphoribosyltransferase domain (Genbank: NP_659489) was released by the "full-length long Japan" (FLJ) collection project at NCBI (Ota et al. 2004). To further characterize its genetic determinants and its function in the salvage pathway we isolated, from the human fetal brain library (Bateman et al. 2004), cDNA encoding uracil phosphoribosyltransferase which belongs to the PRT family (Pfam00156). Here we report the isolation, expression, purification, and characterization of human uracil phosphoribosyltransferase.

\section{Materials and methods}

Cloning and bioinformatic analysis of human UPRTase

A high-quality cDNA library was constructed by using the human fetal brain poly $(\mathrm{A})^{+}$mRNA and a SMART PCR cDNA library construction kit (Clontech). After S $f i$ I digestion, cDNAs greater than $500 \mathrm{bp}$ were ligated into the $\mathrm{S} f i$ I A and S $f$ I B sites of the modified pBluescript II SK $(+)$ vector and then transformed into E. coli $\mathrm{DH} 5 \alpha$ by electroporation (E. coli pulser, Bio-Rad). Both $5^{\prime}$ and $3^{\prime}$ 
ESTs were generated with either dye primer or dye terminator chemistry on an ABI377 sequencer using M13 consensus primers. Primer walking was performed if necessary. Assembly software (Sanger Center) was used to assemble the full-length cDNA sequences. By highthroughput cDNA sequencing a human UPRTase cDNA clone was isolated.

DNA and protein sequence comparisons were performed with BLAST at NCBI (http://www.ncbi.nlm.nih.gov/blast). Multiple sequence alignment analysis was performed with ClustalW algorithms. Protein sorting analysis was done with PSORT II (http://www.psort.nibb.ac.jp:8800). Other sequence analyses were performed with Genedoc software.

\section{RT-PCR of human UPRTase}

To determine the expression profile of the human UPRTase gene, one human multiple tissue cDNA (MTC) panel was used as PCR template in accordance with the manufacturer's procedure (Clontech). The primer sequences of the human UPRTase were 5'-tggtgacagtagcagctatgacgca-3' (human UPRTase-f, corresponding to nucleotides 428-452) and $5^{\prime}$ aggtgcaacaggatgaacttcagtag-3' (human UPRTase-r, corresponding to nucleotides 1051-1076). Twenty-four cycles (for the control, G3PDH) or 32 cycles (for human UPRTase) of amplifications $\left(30 \mathrm{~s}\right.$ at $94^{\circ} \mathrm{C}, 30 \mathrm{~s}$ at $55^{\circ} \mathrm{C}$, and $60 \mathrm{~s}$ at $72^{\circ} \mathrm{C}$ ) were performed using $P f u$ DNA polymerase in $25 \mu \mathrm{L}$. The PCR product of human UPRTase was resolved on $1.5 \%$ Metaphor agarose (Gibco BRL).

Expression, purification, and enzymatic assay of human UPRTase

The open reading frame (ORF) of human UPRTase gene was cloned by PCR using the $P f u$ DNA polymerase. The sequences of forward and reverse synthetic primers were 5'cgggatccgccacggagttacagtgtccgg-3' (BamHI site, underlined) and 5'-cccaagcttttagtctgttccaaagtatttctgtc-3' (HindIII site), respectively. They were designed to anneal to upstream and downstream flanking sequences just outside the human UPRTase and to contain BamHI and HindIII recognition sequences. The 927-bp PCR product was inserted into the $\mathrm{pQE} 30$ vector (Qiagen). Purification of plasmids, agarose gel electrophoresis, and transformation of cells were performed according to the procedures previously described by Maniatis et al. (1982). The recombinant human UPRTase plasmid was transformed into E. coli M15 (pREP4) (Qiagen). The transformed M15 cells were then grown in LuriaBertani (LB) medium (containing $100 \mathrm{mg} \mathrm{mL}^{-1}$ ampicillin and $50 \mathrm{mg} \mathrm{mL}^{-1}$ Kanamycin) at $37^{\circ} \mathrm{C}$ until the culture reached an $\mathrm{OD}_{600}$ of $0.6-0.8$. After $6 \mathrm{~h}$ induction with $0.5 \mathrm{mmol} \mathrm{L}^{-1}$ isopropyl-D-thiogalactopyranoside (IPTG) at $25^{\circ} \mathrm{C}$, cells were harvested by centrifugation and re-sus- pended in cold sodium phosphate buffer $\left(50 \mathrm{mmol} \mathrm{L}^{-1}\right.$ $\mathrm{NaH}_{2} \mathrm{PO}_{4}, \mathrm{pH} \quad 8.0 ; 300 \mathrm{mmol} \mathrm{L}^{-1} \mathrm{NaCl} ; 10 \mathrm{mmol} \mathrm{L}^{-1}$ imidazole), and $0.1 \mathrm{mmol} \mathrm{L}^{-1}$ phenyl methyl sulfonyl fluoride (PMSF) at $10 \mathrm{~mL} \mathrm{~g}^{-1}$ wet weight. The purification steps of human UPRTase were performed by Ni-NTA affinity chromatography as described previously, with slight modification ( $\mathrm{Li}$ et al. 2005). The purified protein was dialyzed against stock buffer containing $5 \mathrm{mmol} \mathrm{L}^{-1}$ Tris- $\mathrm{HCl}$ (pH 8.0), $0.1 \mathrm{mmol} \mathrm{L}^{-1}$ EDTA and 50\% (v/v) glycerol. The purified protein was stored at $-70^{\circ} \mathrm{C}$ until used. The fractions were analyzed by $12 \%$ SDS-PAGE and bands were visualized by Coomassie blue staining by previously described standard procedures. Protein concentrations in extracts and during purification evaluation were determined by the method of Bradford (1976), using bovine serum albumin (BSA) as protein standard.

Human UPRTase activity was determined spectrophotometrically by measuring the conversion of uracil into $\mathrm{UMP}$ at $280 \mathrm{~nm}\left(E\right.$ at $\left.280 \mathrm{~nm}=2.5 \mathrm{mmol}^{-1} \mathrm{~L} \mathrm{~cm}^{-1}\right)$, using a modification of the method of Natalini et al. (1979). PRPP, uracil, UMP, and pyrophosphate sodium were commercial products from Sigma-Aldrich. Reactions were preheated for $5 \mathrm{~min}$ at $37^{\circ} \mathrm{C}$ with PRPP and UPRTase. The reaction mixture of a standard experiment consisted of $0.5 \mathrm{mmol} \mathrm{L}{ }^{-1}$ uracil, $0.5 \mathrm{mmol} \mathrm{L}-1$ PRPP, $10 \mathrm{mmol} \mathrm{L}^{-1}$ $\mathrm{MgCl}_{2}$, and $0.1 \mathrm{mmol} \mathrm{L}^{-1}$ Tris- $\mathrm{HCl}$ buffer $\mathrm{pH} 7.8$, in a final volume of $0.25 \mathrm{~mL}$. The reaction was initiated by addition of an appropriate amount of human UPRTase protein and monitored at $280 \mathrm{~nm}$ by means of an Ultrospec 4000 spectrophotometer (Pharmacia). The reaction was stopped by heating at $100^{\circ} \mathrm{C}$ for $1 \mathrm{~min}$. No reaction was detected in the absence of uracil, PRPP, or enzyme. Kinetic data were analyzed by non-linear regression using the software Origin 6.0 (MicroCal Software).

\section{Subcellular location of human UPRTase}

The ORF of human UPRTase was cloned into pEGFP-C1 expression vector (Clontech) enabling the expression of human UPRTase as a green fluorescent fusion protein (GFP). The sequences of forward and reverse synthetic primers were 5-gaagatctatggccacggagttacagtg-3 (BglII site) and 5-ggggtacccttagtctgttccaaagtattc-3 (KpnI site), respectively. The AD293 cells were maintained in Dulbecco's modified Eagle's medium supplemented with $10 \%$ fetal calf serum, and $100 \mathrm{mg} \mathrm{mL}^{-1}$ ampicillin. Cells were grown at $37^{\circ} \mathrm{C}$ in $5 \% \quad \mathrm{CO}_{2}$. When the growth density of the mammal cells was $70 \%$ complete, the generated fusion plasmid $(2 \mu \mathrm{g} / \mathrm{well})$ was transfected into AD293 cells in a six-well plate with Lipofectamine 2000 (Invitrogen) in accordance with the manufacturer's instructions. After $24 \mathrm{~h}$ transfection the cells were harvested and colored by DAPI for further analyses. 
Phylogenetic tree of human UPRTase

To determine the evolutionary position of human UPRTase in the PRTase family, MEGA software (http:// www.megasoftware.net) was used to construct the phylogenetic tree. The sequences of UPRTases or UPRTasedomain containing proteins from 62 organisms were obtained from NCBI (http://www.ncbi.nlm.nih.gov). Phylogenetic trees for all UPRTase proteins are constructed on the basis of multiple sequence alignments generated with ClustalW using the neighbor-joining algorithm. The trees are then converted into distance matrices. Comparison of these three distance matrices gives information about the evolutionary history of the proteins.

\section{Results and discussion}

Cloning and bioinformatic analysis of human UPRTase

Human UPRTase was isolated from the human fetal brain cDNA library, which encodes a 309 amino acid protein with a molecular mass of approximately $33.8 \mathrm{kDa}$ and a calculated pI of 5.85. The amino acid sequence of human UPRTase is highly conserved and has 97\% identity with Macaca mulatta hypothetical protein (XP_001097557), 86\% identity with Bos taurus (XP_613428), 85\% identity with Rattus norvegicus (XP_001053733), 83\% identity with Canis familiaris (XP_538081), 68\% identity with Danio rerio (NP_996974), 66\% identity with Drosophila

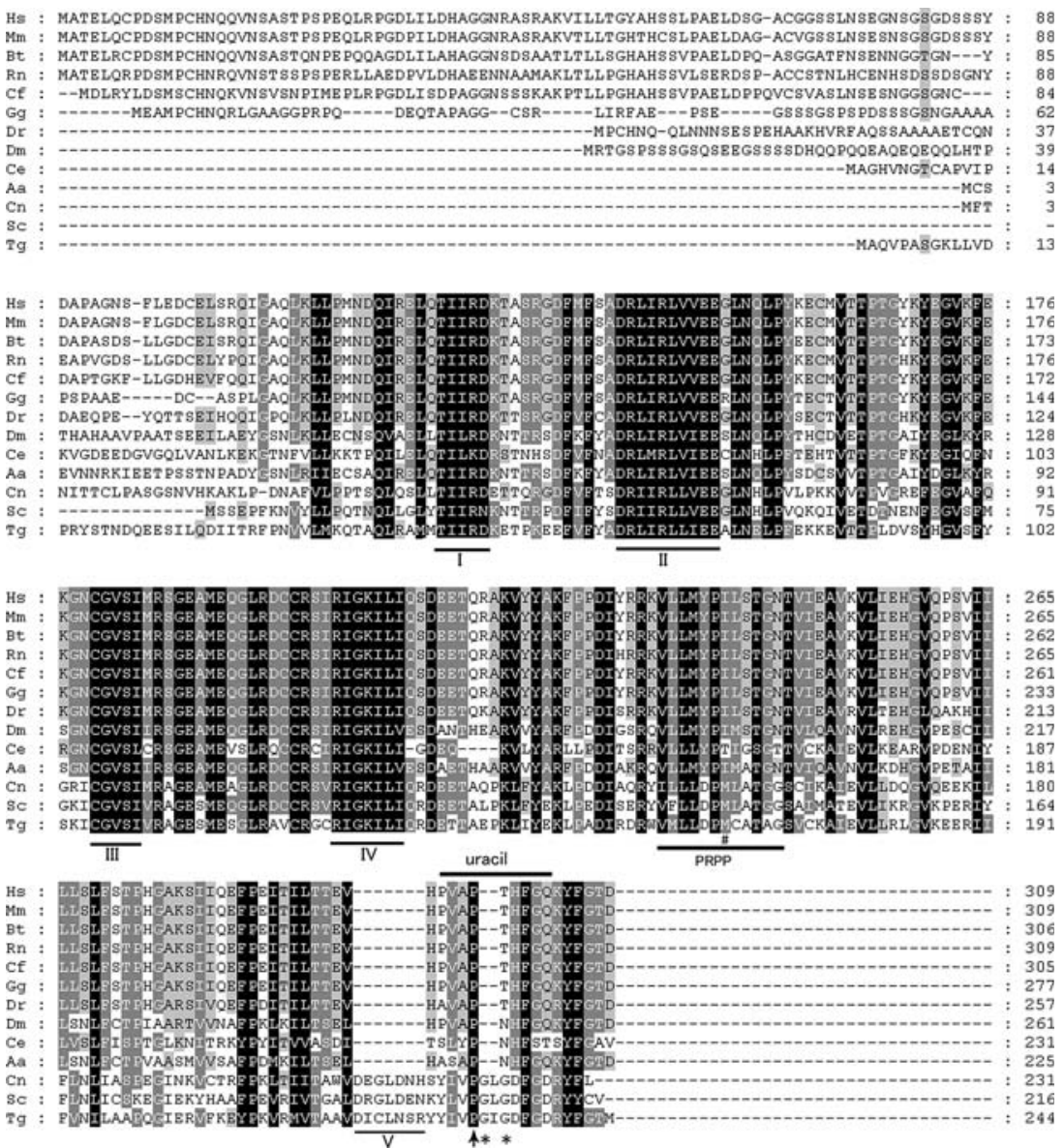

Fig. 1 Protein sequence alignment of human UPRTase ( $H s$, NP_659489), Macaca mulatta (Mm, XP_001097557), Bos taurus (Bt, XP_613428), Rattus norvegicus (Rn, XP_001053733), Canis familiaris (Cf, XP_538081), Gallus gallus ( $G g$, NP_001026295), Danio rerio (Dr, NP_996974), Drosophila melanogaster (Dm, NP_647990), Caenorhabditis elegans (Ce, NP_493268), Aedes aegypti UPRTase (Aa, EAT34814), Cryptococcus neoformans UPRTase (Cn, XP_570874), Saccharomyces cerevisiae UPRTase (Sc, NP_011996), and Toxoplasma gondii UPRTase (Tg,
AAB60213). Conserved amino acids are shaded black. Four short regions $(I-I V)$ had sequence identity. Seven amino acid residues present in prokaryotes are indicated ' $V$ '. The PRPP-binding motif region and the uracil binding region near the C-terminal end are shown by black lines. One proline, highly conserved in the UPRTase family, is represented by an arrow and two glycines are shown with asterisks. Met166, conserved in UPRTases of prokaryotes and important in stacking interactions for PRPP binding, is shown with a hash symbol 


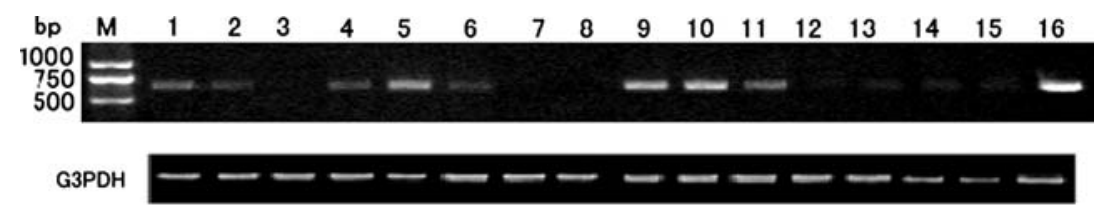

Fig. 2 Reverse transcription PCR analysis of human UPRTase in different human tissues. G3PDH was used as control. 1 Heart, 2 brain, 3 placenta, 4 lung, 5 liver, 6 skeletal muscle, 7 kidney, 8 pancreas, 9

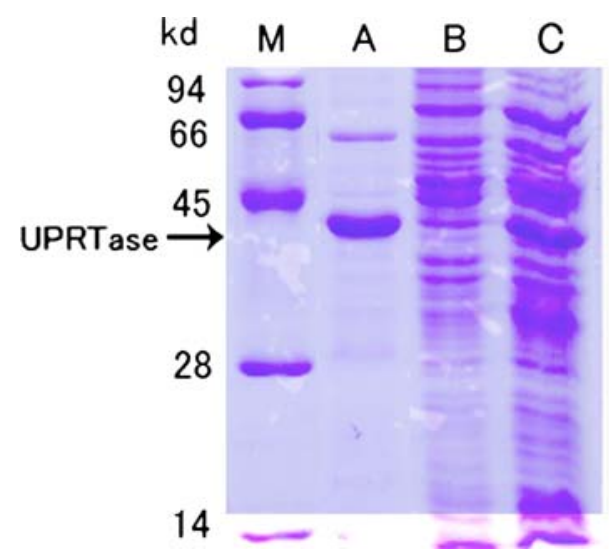

Fig. 3 SDS-PAGE showing expression in E. coli and purification of the recombinant human UPRTase protein. Lane $M$ protein molecular mass standards, Lane A peak fraction from Ni-NTA chromatography, Lane $B$ the supernatant of bacteria after sonication with IPTG induction, and Lane $C$ crude extract from $E$. coli cells induced with IPTG

melanogaster (NP_647990), 65\% identity with Aedes aegypti UPRTase (EAT34814), and similarity with other UPRTases (Fig. 1). By searching the human genome database, human UPRTase was mapped to Xq13.3 in a contig NW_927713.1. The results from this comparison spleen, 10 thymus, 11 prostate, 12 testis, 13 ovary, 14 small intestine, 15 colon, 16 blood leukocytes. The expected size of UPRTase is $650 \mathrm{bp}$

also showed that the human UPRTase gene had seven exons and six introns spanning $30 \mathrm{~kb}$ of human genomic DNA.

Sequence alignments of multiple UPRTase protein sequences reveal a high homology among these enzymes and clearly suggest that their three-dimensional structures will be very similar (Kadziola et al. 2002) (Fig. 1). Four short regions (I-IV) of the UPRTase family encircled the active site in T. gondii UPRTase (Schumacher et al. 1998) and were important to substrate recognition, catalysis, or the stability of the protein. All UPRTases contain a conserved thirteen-residue PRPP-binding motif region, which typically comprised four hydrophobic residues then two acidic residues, two hydrophobic residues, and four small residues, for example glycine (Argos et al. 1983). Near the Cterminal end UPRTases contain a highly conserved twelveamino acid residues sequence that is involved in uracil binding whereas in UPRTases of humans or other mammals two amino acid residues are absent in this region (Fig. 1).

Tissue distribution of human UPRTase

An MTC basic RT-PCR was used to inspect the expression pattern of human UPRTase. The human UPRTase
Fig. 4 Subcellular location of human UPRTase-pEGFP fusion protein in transfected AD293 cells. a pEGFP protein in AD293 cells, $\mathbf{c}$ human UPRTase-pEGFP fusion protein in AD293 cells, $\mathbf{b}$ and $\mathbf{d}$ show the proteins colored with DAPI in a and $\mathbf{c}$, respectively. The human UPRTase-pEGFP fusion protein is distributed in the nucleus and cytoplasm of AD293 cells. The pEGFP control is distributed throughout the whole cell in AD293 cells, as expected

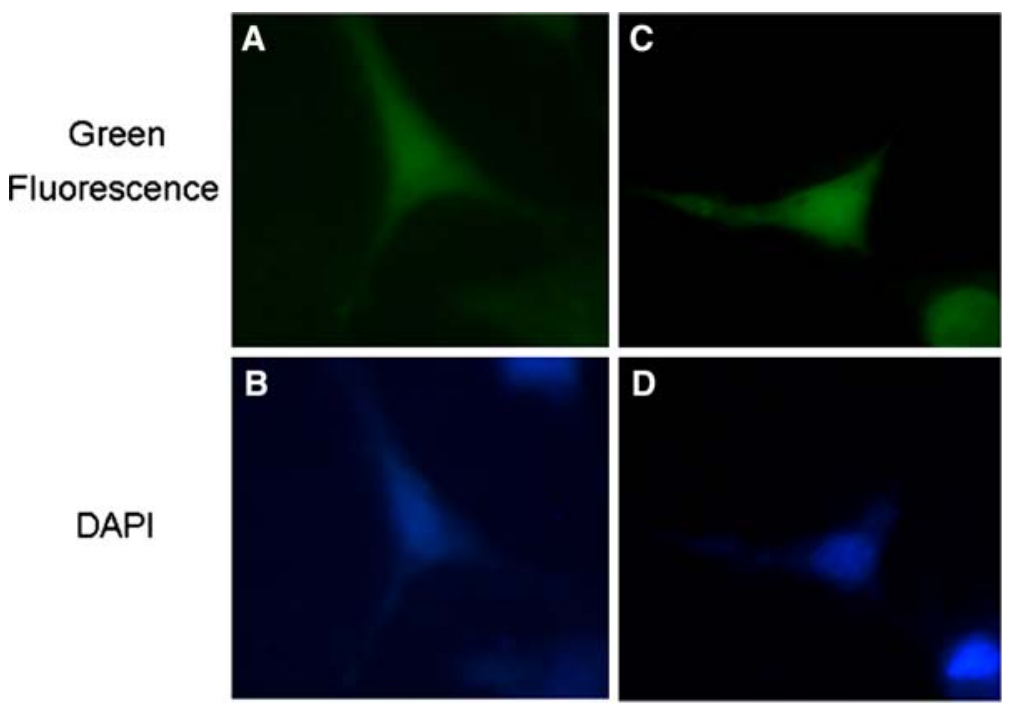


was highly expressed in blood leukocytes, liver, spleen, and thymus, with lower expression levels in prostate, heart, brain, lung, and skeletal muscle; no expression was detected in other tissues (Fig. 2). The size of the product was the expected $650 \mathrm{bp}$. In the PRTase family, adenine phosphoribosyltransferase and hypoxanthine phosphoribosyltransferase are expressed ubiquitously in human tissues (Broderick et al. 1987). This suggests that UPRTase and other PRTases may be important in human tissues.
Expression, purification, and enzymatic assay of human UPRTase

The human UPRTase was cloned into pQE30 and expressed in E. coli M15. The first attempt to purify the recombinant protein, by induction of $E$. coli harboring the construct with IPTG $\left(0.1-1.0 \mathrm{mmol} \mathrm{L}{ }^{-1}\right)$ at $37^{\circ} \mathrm{C}$, failed, because most of the protein expression formed inclusion bodies. Conditions were optimized to increase the solubility of the expressed his-tag UPRTase protein. As shown

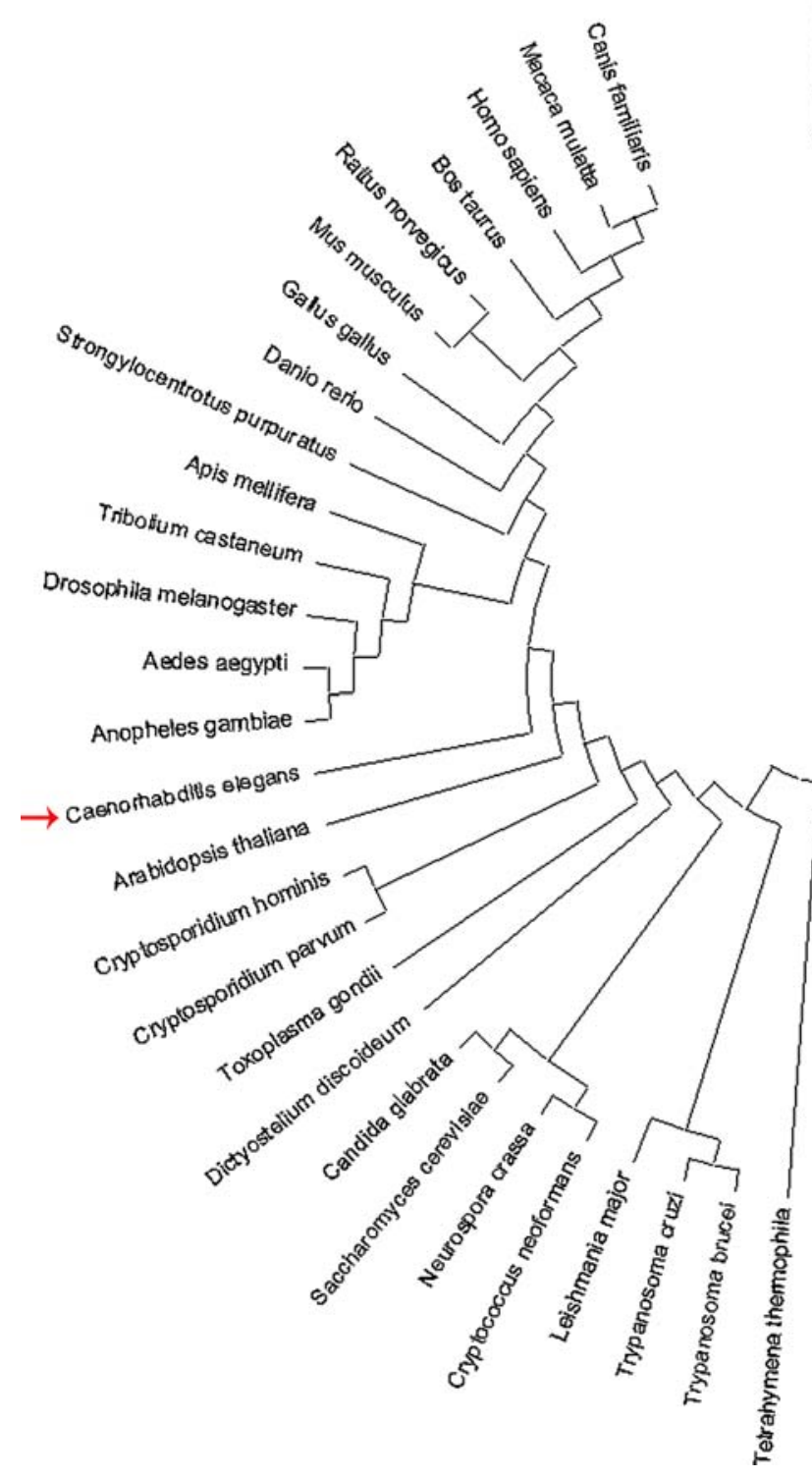

Fig. 5 A uracil phosphoribosyltransferase (UPRTase) family tree based on sequence homology of the conserved UPRTase domain. The known sequences of UPRTases or UPRTase-domain containing

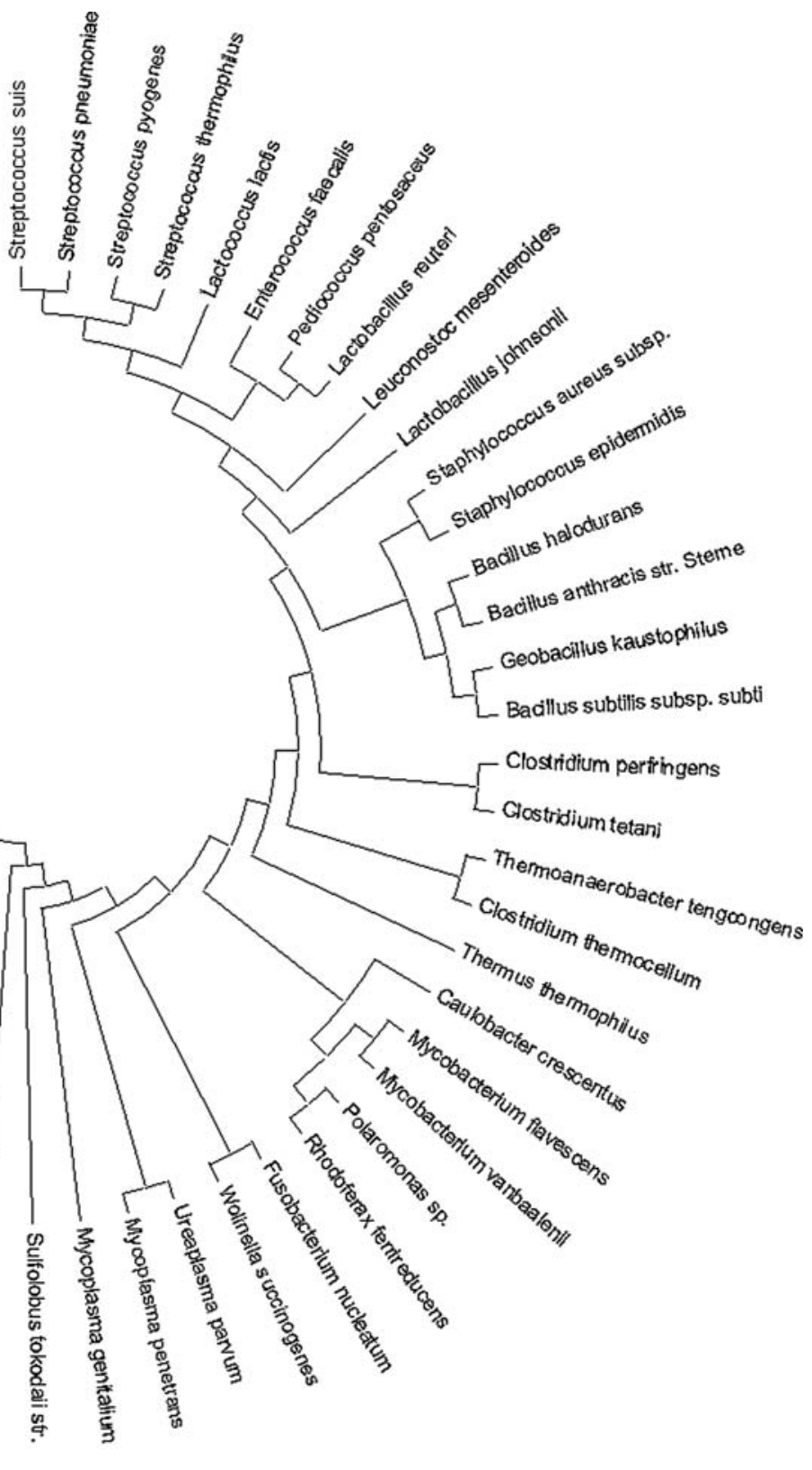

proteins from 62 organisms were aligned by use of ClustalW software. The evolutionary tree was drawn with MEGA software. The red arrow indicates the UPRTase of $C$. elegans 
in Fig. 3, the fusion protein was expressed at $25^{\circ} \mathrm{C}$ on induction with $0.5 \mathrm{mmol} \mathrm{L}^{-1}$ IPTG. The expected $36-\mathrm{kDa}$ fraction was detected by SDS-PAGE, and included one $6 \times$ His tag in the recombinant protein. The soluble recombinant protein was purified by Ni-NTA affinity chromatography to homogeneity. The activity of the human UPRTase was assayed spectrophotometrically at $280 \mathrm{~nm}$. We could not detect UPRTase activity in vitro, however.

Subcellular location of human UPRTase

The human UPRTase-pEGFP fusion plasmid was transfected into AD293 cells. After $24 \mathrm{~h}$ of expression the human UPRTase-pEGFP product was detected by fluorescence microscopy. The results showed that the human UPRTase-pEGFP fusion protein was distributed in the nucleus and cytoplasm of AD293 cells. The pEGFP control was distributed throughout the whole cell in AD293 cells, as expected (Fig. 4).

\section{Evolution analysis of human UPRTase}

The evolution tree of the UPRTase family was constructed from 62 organisms by use of MEGA software (Fig. 5). The result showed that UPRTase selectivity for its pyrimidine substrate was provided solely by backbone contacts and a water-mediated hydrogen bond; one proline and two glycines were critical for the conformation of this region and for uracil binding (Schumacher et al. 1998; Islam et al. (2006). Although in the UPRTases of human and other higher eukaryotes Pro298 is highly conserved, two glycines are absent from the uracil-binding region (Fig. 1). Met166 in T. gondii UPRTase and $B$. subtilis PyrR are also conserved and are important in stacking interactions for PRPP binding (Schumacher et al. 1998), whereas the corresponding site was Ile or Thr in the UPRTases of higher eukaryotes. It was also found that seven amino acid residues (region V, Fig. 1) present in prokaryotes formed one $\beta$-sheet and provided a suitable pocket for uracil binding in $T$. gondii UPRTase. Because UPRTases were conserved from $S$. thermophilus to human, these differences may mean the UPRTases in high eukaryotes have other functions after the divergence from Caenorhabditis elegans.

Although we could not detect UPRTase activity in vitro, it is reasonable to assume human UPRTase has other functions either alone or with other, unknown, factors in vivo, because of its high conservation. To determine UPRTase activity with a more sensitive method, or the potential cofactors, would help to clarify the functions of human UPRTase. Previous research has shown that UPRTase is an attractive target for rational drug design by incorporation of prodrugs that are lethal to many parasitic organisms specifically (Carter et al. 1997; Koyama et al. (2000). This therapeutic strategy must be taken into account carefully and cautiously if the functions of human UPRTase are elucidated clearly. The functions of human UPRTase need further investigation.

Acknowledgments We thank Dr Tonghai Dou and Dr Wenbo Yu of Fudan University for helpful suggestions in relation to this paper. This work was supported by funds from the National Science Foundation of China (Grant Number 10490190 and 10490193).

\section{References}

Alloush HM, Kerridge D (1994) Characterisation of a partially purified uracil phosphoribosyltransferase from the opportunistic pathogen Candida albicans. Mycopathologia 125:129-141

Argos P, Hanei M, Wilson JM, Kelley WN (1983) A possible nucleotide-binding domain in the tertiary fold of phosphoribosyltransferases. J Biol Chem 258:6450-6457

Asai T, Lee CS, Chandler A, O’Sullivan WJ (1990) Purification and characterization of uracil phosphoribosyltransferase from $\mathrm{Cri}$ thidia luciliae. Comp Biochem Physiol B 95:159-163

Bateman A, Coin L, Durbin R, Finn RD, Hollich V, Griffiths-Jones S, Khanna A, Marshall M, Moxon S, Sonnhammer EL, Studholme DJ, Yeats C, Eddy SR (2004) The Pfam protein families database. Nucleic Acids Res 32:D138-D141

Bradford MM (1976) A rapid and sensitive method for the quantification of microgram quantities of protein utilizing the principle of protein-dye binding. Anal Biochem 72:248-254

Broderick TP, Schaff DA, Bertino AM, Dush MK, Tischfield JA, Stambrook PJ (1987) Comparative anatomy of the human APRT gene and enzyme: nucleotide sequence divergence and conservation of a nonrandom $\mathrm{CpG}$ dinucleotide arrangement. Proc Natl Acad Sci USA 84:3349-3353

Carter D, Donald RG, Roos D, Ullman B (1997) Expression, purification, and characterization of uracil phosphoribosyltransferase from Toxoplasma gondii. Mol Biochem Parasitol 87:137144

Dai YP, Lee CS, O’Sullivan WJ (1995) Properties of uracil phosphoribosyltransferase from Giardia intestinalis. Int J Parasitol 25:207-214

Donald RG, Roos DS (1995) Insertional mutagenesis and marker rescue in a protozoan parasite: cloning of the uracil phosphoribosyltransferase locus from Toxoplasma gondii. Proc Natl Acad Sci USA 92:5749-5753

Giffard PM, Rathsam C, Kwan E, Kwan DW, Bunny KL, Koo SP, Jacques NA (1993) The ftf gene encoding the cell-bound fructosyltransferase of Streptococcus salivarius ATCC 25975 is preceded by an insertion sequence and followed by FUR1 and clpP homologues. J Gen Microbiol 139:913-920

Iltzsch MH, Tankersley KO (1994) Structure activity relationship of ligands of uracil phosphoribosyltransferase from Toxoplasma gondii. Biochem Pharmacol 48:781-792

Islam MR, Kim H, Kang SW, Kim JS, Jeong YM, Hwang HJ, Lee SY, Woo JC, Kim SG (2006) Functional characterization of a gene encoding a dual domain for uridine kinase and uracil phosphoribosyltransferase in Arabidopsis thaliana. Plant Mol Biol [Epub ahead of print]

Jensen KF, Mygind B (1996) Different oligomeric states investigated are involved in the allosteric behaviour of uracil phosphoribosyltransferase from Escherichia coli. Eur J Biochem 204:637645 
Jensen KF, Arent S, Larsen S, Schack L (2005) Allosteric properties of the GTP activated and CTP inhibited uracil phosphoribosyltransferase from the thermoacidophilic archaeon Sulfolobus solfataricus. FEBS J 272:1440-1453

Kadziola A, Neuhard J, Larsen S (2002) Structure of product-bound Bacillus caldolyticus uracil phosphoribosyltransferase confirms ordered sequential substrate binding. Acta Cryst D D58:936-945

Kern L, de Montigny J, Jund R, Lacroute F (1990) The FUR1 gene of Saccharomyces cerevisiae: cloning, structure and expression of wild-type and mutant alleles. Gene 88:149-157

Koyama F, Sawada H, Fuji H, Hamada H, Hirao T, Ueno M, Nakano H (2000) Adenoviral-mediated transfer of Escherichia coli uracil phosphoribosyltransferase (UPRT) gene to modulate the sensitivity of the human colon cancer cells to 5-fluorouracil. Eur J Cancer 36:2403-2410

Kukimoto-Niino M, Shibata R, Murayama K, Hamana H, Nishimoto M, Bessho Y, Terada T, Shirouzu M, Kuramitsu S, Yokoyama S (2005) Crystal structure of a predicted phosphoribosyltransferase (TT1426) from Thermus thermophilus HB8 at 2.01 A resolution. Protein Sci 14:823-827

Li J, Ji C, Chen J, Yang Z, Wang Y, Fei X, Zheng M, Gu X, Wen G, Xie Y, Mao Y (2005) Identification and characterization of a novel Cut family cDNA that encodes human copper transporter protein CutC. Biochem Biophys Res Commun 337:179-183

Linde L, Jensen KF (1996) Uracil phosphoribosyltransferase from the extreme thermoacidophilic archaebacterium Sulfolobus shibatae is an allosteric enzyme, activated by GTP and inhibited by CTP. Biochem Biophys Acta 1296:16-22

Lundegaard C, Jensen KF (1999) Kinetic mechanism of uracil phosphoribosyltransferase from Escherichia coli and catalytic importance of the conserved proline in the PRPP binding site. Biochemistry 38:3327-3334

Magni G, Natalini P, Ruggieri S, Vita A (1976) Bakers' yeast uridine nucleosidase is a regulatory copper containing protein. Biochem Biophys Res Commun 69:724-730

Maniatis T, Fritsch EF, Sambroch J (1982) Molecular cloning. Cold Spring Harbor Laboratories, Cold Spring Harbor, New York, pp $1-188$

Martinussen J, Hammer K (1994) Cloning and characterization of upp, a gene encoding uracil phosphoribosyltransferase from Lactococcus lactis. J Bacteriol 176:6457-6463
Martinussen J, Glaser P, Andersen PS, Saxild HH (1995) Two genes encoding uracil phosphoribosyltransferase are present in Bacillus subtilis. J Bacteriol 177:271-274

McIvor RS, Wohlhueter RM, Plagemann PG (1983) Uracil phosphoribosyltransferase from Acholeplasma laidlawii: partial purification and kinetic properties. J Bacteriol 156:192-197

Musick WD (1981) Structural features of the phosphoribosyltransferases and their relationship to the human deficiency disorders of purine and pyrimidine metabolism. CRC Crit Rev Biochem $11: 1-34$

Natalini P, Ruggieri S, Santarelli I, Vita A, Magni G (1979) Baker's yeast UMP: pyrophosphate phosphoribosyltransferase. Purification, enzymatic and kinetic properties. J Biol Chem 254:15581563

Neuhard J, Kelln RA (1996) In: Neidhart FC, Ingraham JL, Low KB, Magasanik B, Schaechter M, Umbarger HE (eds) Escherichia coli and Salmonella typhimurium: cellular and molecular biology. American Society for Microbiology, Washington

Ota T, Suzuki Y, Nishikawa T et al (2004) Complete sequencing and characterization of 21,243 full-length human cDNAs. Nat Genet 36:40-45

Pfefferkorn ER, Eckel ME, McAdams E (1989) Toxoplasma gondii: the biochemical basis of resistance to emimycin. Exp Parasitol 69:129-139

Schumacher MA, Carter D, Scott DM, Roos DS, Ullman B, Brennan RG (1998) Crystal structures of Toxoplasma gondii uracil phosphoribosyltransferase reveal the atomic basis of pyrimidine discrimination and prodrug binding. EMBO J 17:3219-3232

Schumacher MA, Bashor CJ, Song MH, Otsu K, Zhu S, Parry RJ, Ullman B, Brennan RG (2002) The structural mechanism of GTP stabilized oligomerization and catalytic activation of the Toxoplasma gondii uracil phosphoribosyltransferase. Proc Natl Acad Sci USA 99:78-83

Sinha SC, Smith JL (2001) The PRT protein family. Curr Opin Struct Biol 11:733-739

Traut TW, Jones ME (1996) Uracil metabolism UMP synthesis from orotic acid or uridine and conversion of uracil to a-alanine: enzymes and cDNAs. Prog Nucleic Acid Res Mol Biol 53:1-78 